\title{
Hypothermia, shivering, and dexmedetomidine
}

\author{
Sung Mi Hwang
}

Department of Anesthesiology and Pain Medicine, Chuncheon Sacred Heart Hospital, Hallym University College of Medicine, Chuncheon, Korea

Postoperative temperature is increasingly used to indicate the quality of anesthesia. Anesthesiologists should measure and record the body temperature of their patients perioperatively and maintain it within normal limits. Hypothermia is usually defined as a body temperature less than $36^{\circ} \mathrm{C}$ and occurs commonly during surgery [1]. Although hypothermia can be protective during cerebral or cardiac ischemia, unintentional perioperative hypothermia is associated with an increased mortality rate. To prevent hypothermia during surgery, several methods are used. In this issue of the Korean Journal of Anesthesiology, Kim et al. [2] compared the effect of a forced-air warming system and circulating-water mattress on core temperature and the incidence of postanesthesia shivering in elderly patients. They found that the changes in core temperature over time did not differ between the two types of warming device. However, the incidence of postanesthesia shivering was different (13.0 vs $43.5 \%$ ). The forced-air warming system is more effective in terms of reducing the incidence of postanesthesia shivering. Here, we postulate that the cause of postanesthesia shivering is not confined to hypothermia. De Witte et al. [3] evaluated the efficacy of resistiveheating or forced air warming versus no prewarming, applied before inducing anesthesia, and recommended that prewarming before the induction of anesthesia should be considered as part ofanesthetic management.

Although the cause of postoperative shivering is not clear, most shivering associated with neuraxial anesthesia seems to be normal shivering, the expected response to hypothermia [4]. Perioperative hypothermia and shivering are associated with many adverse perioperative outcomes, including delayed anesthetic recovery, increased blood loss, and surgical wound infection. Furthermore, excessive shivering increases oxygen consumption, and lactic acidosis, and it increases the metabolic rate by up to $400 \%[1,4]$. Neuraxial anesthesia and old age both predict postanesthesia shivering. Therefore preventing shivering is important, especially in elderly patients undergoing neuraxial anesthesia for surgery. Various drugs have been investigated with a view to preventing or treating postoperative shivering. However, the ideal drug remains elusive. Recent reports indicate that dexmedetomidine has good anti-shivering properties [4-7]. In this issue of the Korean Journal of Anesthesiology, Park et al. [8] compared two doses of intravenous dexmedetomidine in elderly patients during spinal anesthesia. They observed the effect of dexmedetomidine on the prolongation of sensory and motor block, but did not monitor body temperature and did not describe a method for maintaining normothermia. However, shivering did not occur in any of the enrolled patients. Consequently, they could not evaluate the anti-shivering effect of dexmedetomidine. Although both Kim et al. [2] and Park et al. [8] studied elderly patients undergoing spinal anesthesia, the incidence of shivering was different. The cause of shivering is not clear. Indeed, many factors can cause shivering, and the incidence varies.

Core temperature monitoring remains rare during regional anesthesia, and hypothermia often goes undetected [4]. In the present aging society, the number of elderly patients who require anesthesia for surgery is increasing. Elderly patients are prone to hypothermia and shivering during the perioperative period. Therefore, both temperature monitoring, and prevention of postoperative shivering are essential during regional or general anesthesia in elderly patients.

Corresponding author: Sung Mi Hwang, M.D., Department of Anesthesiology and Pain Medicine, Chuncheon Sacred Heart Hospital, Hallym University College of Medicine, 77, Sakju-ro, Chuncheon 200-704, Korea. Tel: 82-33-240-5594, Fax: 82-33-251-0941, E-mail: h70sm@hallym.or.kr (c) This is an open-access article distributed under the terms of the Creative Commons Attribution Non-Commercial License (http:// creativecommons.org/licenses/by-nc/3.0/), which permits unrestricted non-commercial use, distribution, and reproduction in any medium, provided the original work is properly cited. 


\section{References}

1. Reynolds L, Beckmann J, Kurz A. Perioperative complications of hypothermia. Best Pract Res Clin Anaesthesiol 2008; 22: 645-57.

2. Kim HY, Lee KC, Lee MJ, Kim MN, Kim JS, Lee WS, et al. Comparison of the efficacy of a forced-air warming system and circulating-water mattress on core temperature and post-anesthesia shivering in elderly patients undergoing total knee arthroplasty under spinal anesthesia. Korean J Anesthesiol 2014; 66: 352-7.

3. De Witte JL, Demeyer C, Vandemaele E. Resistive-heating or forced-air warming for the prevention of redistribution hypothermia. Anesth Analg 2010; 110: 829-33.

4. Sessler DI. Temperature monitoring and perioperative thermoregulation. Anesthesiology 2008; 109: 318-38.

5. Kim YS, Kim YI, Seo KH, Kang HR. Optimal dose of prophylactic dexmedetomidine for preventing postoperative shivering. Int J Med Sci 2013; 10: 1327-32.

6. Usta B, Gozdemir M, Demircioglu RI, Muslu B, Sert H, Yaldiz A. Dexmedetomidine for the prevention of shivering during spinal anesthesia. Clinics (Sao Paulo) 2011; 66: 1187-91.

7. Bicer C, Esmaoglu A, Akin A, Boyaci A. Dexmedetomidine and meperidine prevent postanesthetic shivering. Eur J Anaesthesiol 2006; 23: 149-53.

8. Park SH, Shin YD, Yu HJ, Bae JH, Yim KH. Comparison of two dosing schedules of intravenous dexmedetomidine in elderly patients during spinal anesthesia. Korean J Anesthesiol 2014; 66: 371-6. 